Kragujevac Journal of Mathematics

Volume 41(2) (2017), Pages 179-201.

\title{
SUPER MEAN LABELING OF SOME SUBDIVISION GRAPHS
}

\author{
R. VASUKI, P. SUGIRTHA AND J. VENKATESWARI
}

ABstract. Let $G$ be a graph and $f: V(G) \rightarrow\{1,2,3, \ldots, p+q\}$ be an injection. For each edge $e=u v$, the induced edge labeling $f^{*}$ is defined as follows:

$$
f^{*}(e)= \begin{cases}\frac{f(u)+f(v)}{2}, & \text { if } f(u)+f(v) \text { is even, } \\ \frac{f(u)+f(v)+1}{2}, & \text { if } f(u)+f(v) \text { is odd. }\end{cases}
$$

Then $f$ is called super mean labeling if $f(V(G)) \cup\left\{f^{*}(e): e \in E(G)\right\}=$ $\{1,2,3, \ldots, p+q\}$. A graph that admits a super mean labeling is called super mean graph. In this paper, we have studied the super meanness property of the subdivision of the $H$-graph $H_{n}, H_{n} \odot K_{1}, H_{n} \odot S_{2}$, slanting ladder, $T_{n} \odot K_{1}, C_{n} \odot K_{1}$ and $C_{n} @ C_{m}$.

\section{INTRODUCTION}

Throughout this paper, by a graph we mean a finite, undirected and simple graph. Let $G(V, E)$ be a graph with $p$ vertices and $q$ edges. For notations and terminology we follow [2].

The path on $n$ vertices is denoted by $P_{n}$ and a cycle on $n$ vertices is denoted by $C_{n}$. A triangular snake is obtained from a path by identifying each edge of the path with an edge of the cycle $C_{3}$. The graph $C_{m} @ C_{n}$ is obtained by identifying an edge of $C_{m}$ with an edge of $C_{n}$. The slanting ladder $S L_{n}$ is a graph obtained from two paths $u_{1} u_{2} \ldots u_{n}$ and $v_{1} v_{2} \ldots v_{n}$ by joining each $u_{i}$ with $v_{i+1}, 1 \leq i \leq n-1$. The $H$-graph of a path $P_{n}$, denoted by $H_{n}$ is the graph obtained from two copies of $P_{n}$ with vertices $v_{1}, v_{2}, \ldots, v_{n}$ and $u_{1}, u_{2}, \ldots, u_{n}$ by joining the vertices $v_{\frac{n+1}{2}}$ and $u_{\frac{n+1}{2}}$ if $n$ is odd and the vertices $v_{\frac{n}{2}+1}$ and $u_{\frac{n}{2}}$ if $n$ is even. The corona of a graph $G$ on $p$ vertices $v_{1}, v_{2}, \ldots, v_{p}$ is the graph obtained from $G$ by adding $p$ new vertices $u_{1}, u_{2}, \ldots, u_{p}$

Key words and phrases. Super mean graph, super mean labeling.

2010 Mathematics Subject Classification. Primary: 05C78.

Received: November 27, 2014.

Accepted: September 9, 2016. 
and the new edges $u_{i} v_{i}$ for $1 \leq i \leq p$. The corona of $G$ is denoted by $G \odot K_{1}$. The 2-corona of a graph $G$, denoted by $G \odot S_{2}$ is a graph obtained from $G$ by identifying the center vertex of the star $S_{2}$ at each vertex of $G$. A graph which can be obtained from a given graph by breaking up each edge into one or more segments by inserting intermediate vertices between its two ends. If each edge of a graph $G$ is broken into two by exactly one vertex, then the resultant graph is taken as $S(G)$.

A vertex labeling of $G$ is an assignment $f: V(G) \rightarrow\{1,2, \ldots, p+q\}$ be an injection. For a vertex labeling $f$, the induced edge labeling $f^{*}(e=u v)$ is defined by

$$
f^{*}(e)= \begin{cases}\frac{f(u)+f(v)}{2}, & \text { if } f(u)+f(v) \text { is even, } \\ \frac{f(u)+f(v)+1}{2}, & \text { if } f(u)+f(v) \text { is odd. }\end{cases}
$$

Then $f$ is called super mean labeling if

$$
f(V(G)) \cup\left\{f^{*}(e): e \in E(G)\right\}=\{1,2,3, \ldots, p+q\} .
$$

Clearly $f^{*}$ is injective. A graph that admits a super mean labeling is called super mean graph.

A super mean labeling of the graph $P_{7}^{2}$ is shown in Figure 1.

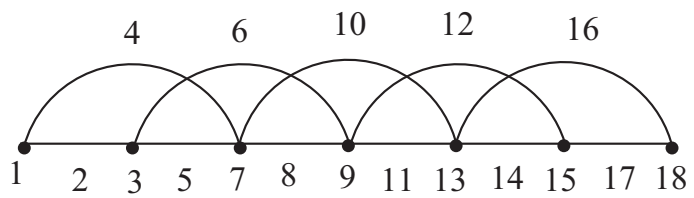

FIGURE 1

The concept of mean labeling was introduced and studied by S. Somasundaram and R. Ponraj [5]. Some new families of mean graphs are discussed in [10,11].

The concept of super mean labeling was introduced and studied by D. Ramya et al. [4]. Further some more results on super mean graphs are discussed in [1,3,6-9].

In this paper, we have studied the super meanness of the subdivision of the graphs $H$-graph $H_{n}, H_{n} \odot K_{1}, H_{n} \odot S_{2}$, slanting ladder, $T_{n} \odot K_{1}, C_{n} \odot K_{1}$ and $C_{n} @ C_{m}$.

\section{Super Mean Graphs}

Theorem 2.1. The graph $S\left(H_{n}\right)$ is a super mean graph, for $n \geq 3$.

Proof. Let $u_{1}, u_{2}, \ldots, u_{n}$ and $v_{1}, v_{2}, \ldots, v_{n}$ be the vertices of the paths of length $n-1$. Each edge $u_{i} u_{i+1}$ is subdivided by a vertex $x_{i}, 1 \leq i \leq n-1$ and each edge $v_{i} v_{i+1}$ is subdivided by a vertex $y_{i}, 1 \leq i \leq n-1$. The edge $u_{\frac{n+1}{2}} v_{\frac{n+1}{2}}$ is divided by a vertex $z$ when $n$ is odd. The edge $u_{\frac{n+2}{2}} v_{\frac{n}{2}}$ is divided by a vertex $z$ when $n$ is even. The graph $S\left(H_{n}\right)$ has $4 n-1$ vertices and $4 n-2$ edges.

Define $f: V\left(S\left(H_{n}\right)\right) \rightarrow\{1,2,3, \ldots, p+q=8 n-3\}$ as follows:

$$
f\left(u_{i}\right)=4 i-3, \quad 1 \leq i \leq n,
$$




$$
\begin{aligned}
& f\left(v_{i}\right)= \begin{cases}4(n+i)-5, & 1 \leq i \leq\left\lfloor\frac{n-1}{2}\right\rfloor, \\
4(n+i)-3, & \left\lfloor\frac{n+1}{2}\right\rfloor \leq i \leq n,\end{cases} \\
& f\left(x_{i}\right)=4 i-1, \quad 1 \leq i \leq n-1, \\
& f\left(y_{i}\right)= \begin{cases}4(n+i)-3, & 1 \leq i \leq\left\lfloor\frac{n-1}{2}\right\rfloor, \\
4(n+i)-1, & \left\lfloor\frac{n+1}{2}\right\rfloor \leq i \leq n-1,\end{cases} \\
& \text { and } f(z)= \begin{cases}6 n-4, & \text { if } n \text { is odd, } \\
6 n-6, & \text { if } n \text { is even }\end{cases}
\end{aligned}
$$

For the vertex labeling $f$, the induced edge labeling is given as follows:

$$
\begin{aligned}
& f^{*}\left(u_{i} x_{i}\right)=4 i-2, \quad 1 \leq i \leq n-1, \\
& f^{*}\left(x_{i} u_{i+1}\right)=4 i, \quad 1 \leq i \leq n-1 \text {, } \\
& f^{*}\left(v_{i} y_{i}\right)= \begin{cases}4(n+i)-4, & 1 \leq i \leq\left\lfloor\frac{n-1}{2}\right\rfloor, \\
4(n+i)-2, & \left\lfloor\frac{n+1}{2}\right\rfloor \leq i \leq n-1,\end{cases} \\
& f^{*}\left(y_{i} v_{i+1}\right)= \begin{cases}4(n+i)-2, & 1 \leq i \leq\left\lfloor\frac{n-3}{2}\right\rfloor \\
6 n-3, & i=\frac{n-1}{2} \text { and } n \text { is odd } \\
6 n-5, & i=\frac{n-2}{2} \text { and } n \text { is even } \\
4(n+i), & \left\lfloor\frac{n+1}{2}\right\rfloor \leq i \leq n-1\end{cases} \\
& f^{*}\left(u_{\frac{n+1}{2}} z\right)=4 n-2, \quad \text { if } n \mathrm{rm} \text { is odd, } \\
& f^{*}\left(z v_{\frac{n+1}{2}}\right)=6 n-2, \quad \text { if } n \text { is odd, } \\
& f^{*}\left(u_{\frac{n+2}{2}} z\right)=4 n-2, \quad \text { if } n \text { is even, } \\
& \text { and } f^{*}\left(z v_{\frac{n}{2}}\right)=6 n-4, \quad \text { if } n \text { is even. }
\end{aligned}
$$

Thus, $f$ is a super mean labeling and hence $S\left(H_{n}\right)$ is a super mean graph.

For example, a super mean labeling of $S\left(H_{7}\right)$ and $S\left(H_{8}\right)$ are shown in Figure 2.

Theorem 2.2. The graph $S\left(H_{n} \odot K_{1}\right)$ is a super mean graph, for $n \geq 3$.

Proof. Let $u_{1}, u_{2}, \ldots, u_{n}$ and $v_{1}, v_{2}, \ldots, v_{n}$ be the vertices of the paths of length $n-1$. Let $a_{1, i} a_{2, i} u_{i}$ be the path attached at each $u_{i}, 1 \leq i \leq n$ and $b_{1, i} b_{2, i} v_{i}$ be the path attached at each $v_{i}, 1 \leq i \leq n$. Each edge $u_{i} u_{i+1}$ is subdivided by a vertex $x_{i}$, $1 \leq i \leq n-1$ and each edge $v_{i} v_{i+1}$ is subdivided by a vertex $y_{i}, 1 \leq i \leq n-1$. The edge $u_{\frac{n+1}{2}} v_{\frac{n+1}{2}}$ is divided by a vertex $z$ when $n$ is odd. The edge $u_{\frac{n+2}{2}} v_{\frac{n}{2}}$ is divided by a vertex $z$ when $n$ is even. The graph $S\left(H_{n} \odot K_{1}\right)$ has $8 n-1$ vertices and $8 n-2$ edges. 


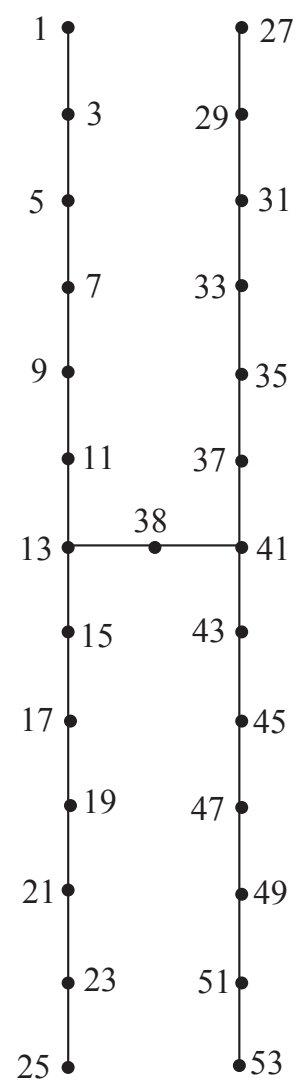

(a) $S\left(H_{7}\right)$

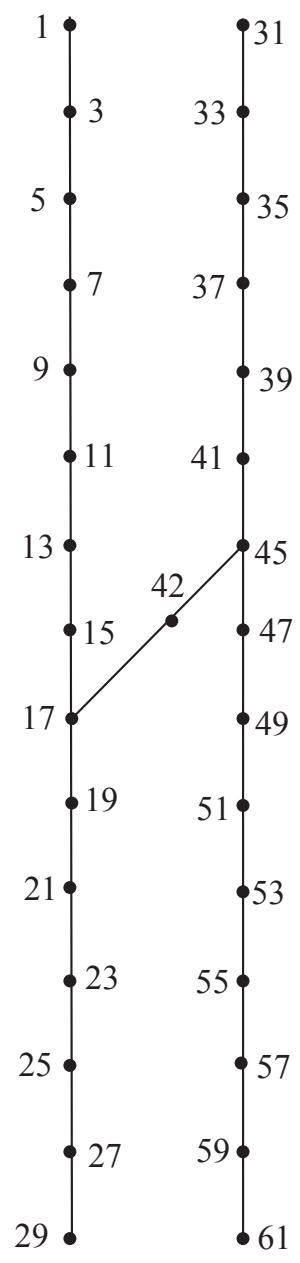

(b) $S\left(H_{8}\right)$

Figure 2

Define $f: V\left(S\left(H_{n} \odot K_{1}\right)\right) \rightarrow\{1,2,3, \ldots, p+q=16 n-3\}$ as follows:

$$
\begin{aligned}
f\left(u_{i}\right) & = \begin{cases}5, & i=1, \\
8 i-7, & 2 \leq i \leq n,\end{cases} \\
f\left(v_{i}\right) & = \begin{cases}8 n+3, & i=1, \\
8(n+i)-9, & 2 \leq i \leq\left\lfloor\frac{n-1}{2}\right\rfloor, \\
8(n+i)-7, & \left\lfloor\frac{n+1}{2}\right\rfloor \leq i \leq n,\end{cases} \\
f\left(a_{1, i}\right) & = \begin{cases}1, & i=1, \\
8 i-2, & 2 \leq i \leq n,\end{cases} \\
f\left(a_{2, i}\right) & =8 i-5, \quad 1 \leq i \leq n .
\end{aligned}
$$




$$
\begin{aligned}
& f\left(b_{1, i}\right)= \begin{cases}8 n-1, & i=1, \\
8(n+i)-4, & 2 \leq i \leq\left\lfloor\frac{n-1}{2}\right\rfloor, \\
8(n+i)-2, & \left\lfloor\frac{n+1}{2}\right\rfloor \leq i \leq n-1, \\
16 n-3, & i=n,\end{cases} \\
& f\left(b_{2, i}\right)= \begin{cases}8(n+i)-7, & 1 \leq i \leq\left\lfloor\frac{n-1}{2}\right\rfloor, \\
8(n+i)-5, & \left\lfloor\frac{n+1}{2}\right\rfloor \leq i \leq n\end{cases} \\
& f\left(x_{i}\right)=8 i-1, \quad 1 \leq i \leq n-1, \\
& f\left(y_{i}\right)= \begin{cases}8(n+i)-3, & 1 \leq i \leq\left\lfloor\frac{n-1}{2}\right\rfloor, \\
8(n+i)-1, & \left\lfloor\frac{n+1}{2}\right\rfloor \leq i \leq n-1,\end{cases} \\
& \text { and } f(z)= \begin{cases}12 n-6, & \text { if } n \text { is odd } \\
12 n-10, & \text { if } n \text { is even. }\end{cases}
\end{aligned}
$$

The induced edge labeling is obtained as follows:

$$
\begin{aligned}
& f^{*}\left(u_{i} x_{i}\right)= \begin{cases}6, & i=1, \\
8 i-4, & 2 \leq i \leq n-1,\end{cases} \\
& f^{*}\left(x_{i} u_{i+1}\right)=8 i, \quad 1 \leq i \leq n-1,
\end{aligned}
$$




$$
\begin{aligned}
f^{*}\left(b_{2, i} v_{i}\right) & = \begin{cases}8 n+2, & i=1, \\
8(n+i)-8, & 2 \leq i \leq\left\lfloor\frac{n-1}{2}\right\rfloor, \\
8(n+i)-6, & \left\lfloor\frac{n+1}{2}\right\rfloor \leq i \leq n,\end{cases} \\
f^{*}\left(u_{\frac{n+1}{2}} z\right) & =8 n-4, \quad \text { if } n \text { is odd, } \\
f^{*}\left(z v_{\frac{n+1}{2}}\right) & =12 n-4, \quad \text { if } n \text { is odd. } \\
f^{*}\left(u_{\frac{n+2}{2}} z\right) & =8 n-4, \quad \text { if } n \text { is even, } \\
f^{*}\left(z v_{\frac{n}{2}}\right) & =12 n-8, \quad \text { if } n \text { is even. }
\end{aligned}
$$

Thus, $f$ is a super mean labeling and hence $S\left(H_{n} \odot K_{1}\right)$ is a super mean graph.

For example, a super mean labeling of $S\left(H_{9} \odot K_{1}\right)$ and $S\left(H_{10} \odot K_{1}\right)$ are shown in Figure 3.

Theorem 2.3. The graph $S\left(H_{n} \odot S_{2}\right)$ is a super mean graph, for $n \geq 3$.

Proof. Let $u_{1}, u_{2}, \ldots, u_{n}$ and $v_{1}, v_{2}, \ldots, v_{n}$ be the vertices of the paths of length $n-1$. Let $a_{1, i} a_{2, i} u_{i}$ and $a_{3, i} a_{4, i} u_{i}$ be the paths attached at each $u_{i}, 1 \leq i \leq n$ and $b_{1, i} b_{2, i} v_{i}$ and $b_{3, i} b_{4, i} v_{i}$ be the paths attached at each $v_{i}, 1 \leq i \leq n$. Each edge $u_{i} u_{i+1}$ is subdivided by a vertex $x_{i}, 1 \leq i \leq n-1$ and each edge $v_{i} v_{i+1}$ is subdivided by a vertex $y_{i}$, $1 \leq i \leq n-1$. The edge $u_{\frac{n+1}{2}} v_{\frac{n+1}{2}}$ is divided by a vertex $z$ when $n$ is odd. The edge $u_{\frac{n+2}{2}} v_{\frac{n}{2}}$ is divided by a vertex $z$ when $n$ is even. The graph $S\left(H_{n} \odot S_{2}\right)$ has $12 n-1$ vertices and $12 n-2$ edges.

Define $f: V\left(S\left(H_{n} \odot S_{2}\right)\right) \rightarrow\{1,2,3, \ldots, p+q=24 n-3\}$ as follows:

$$
\begin{aligned}
f\left(u_{i}\right) & =12 i-7, \quad 1 \leq i \leq n, \\
f\left(v_{i}\right) & = \begin{cases}12(n+i)-9, & 1 \leq i \leq\left\lfloor\frac{n-1}{2}\right\rfloor, \\
12(n+i)-7, & \left\lfloor\frac{n+1}{2}\right\rfloor \leq i \leq n,\end{cases} \\
f\left(a_{1, i}\right) & = \begin{cases}1, & i=1, \\
12 i-13, & 2 \leq i \leq n,\end{cases} \\
f\left(a_{2, i}\right) & = \begin{cases}3, & i=1, \\
12 i-11, & 2 \leq i \leq n,\end{cases} \\
f\left(a_{3, i}\right) & =12 i-3, \quad 1 \leq i \leq n, \\
f\left(a_{4, i}\right) & =12 i-5, \quad 1 \leq i \leq n, \\
f\left(x_{i}\right) & =12 i+2, \quad 1 \leq i \leq n-1,
\end{aligned}
$$




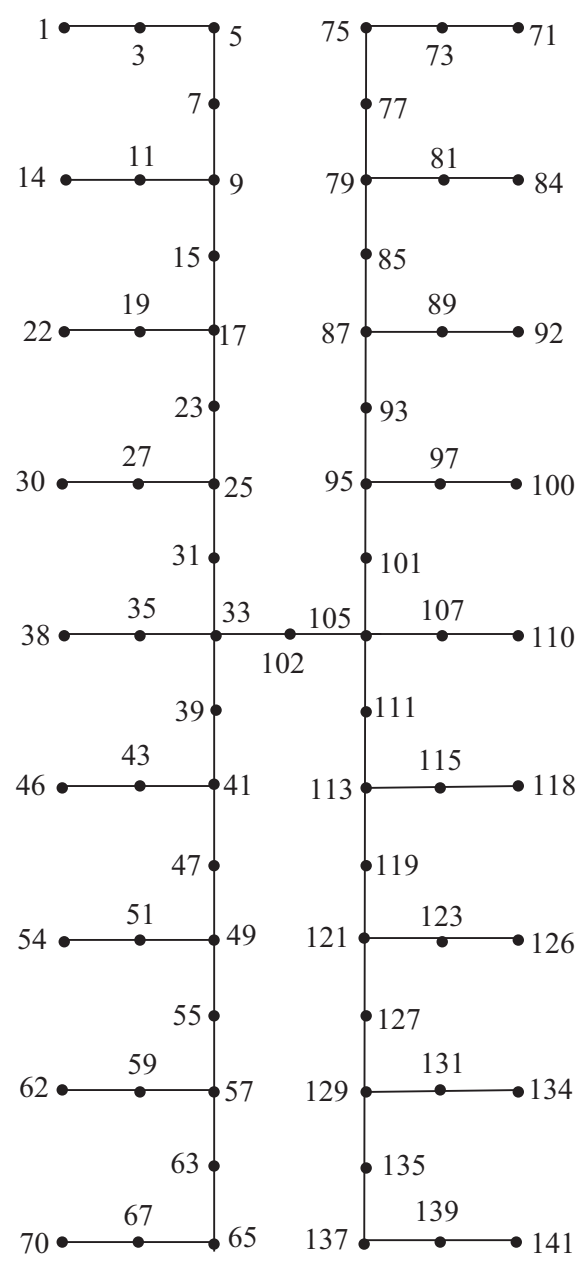

(a) $S\left(H_{9} \odot K_{1}\right)$

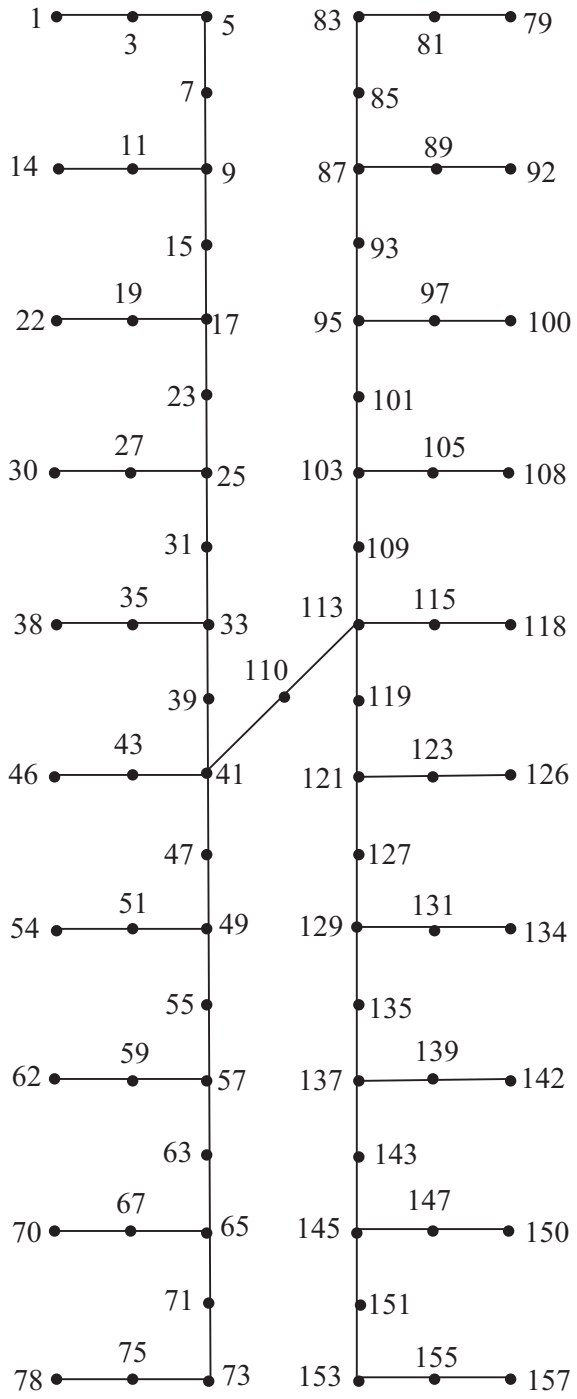

(b) $S\left(H_{1} 0 \odot K_{1}\right)$

FiguRE 3

$$
f\left(b_{1, i}\right)= \begin{cases}12 n-1, & i=1 \\ 12(n+i)-15, & 2 \leq i \leq\left\lfloor\frac{n-1}{2}\right\rfloor \\ 18 n-8, & i=\frac{n+1}{2} \text { and } n \text { is odd } \\ 18 n-14, & i=\frac{n}{2} \text { and } n \text { is even } \\ 12(n+i)-13, & \left\lfloor\frac{n+3}{2}\right\rfloor \leq i \leq n\end{cases}
$$




$$
\begin{aligned}
& f\left(b_{2, i}\right)= \begin{cases}12 n+1, & i=1 \\
12(n+i)-13, & 2 \leq i \leq\left\lfloor\frac{n-1}{2}\right\rfloor \\
18 n-6, & i=\frac{n+1}{2} \text { and } n \text { is odd } \\
18 n-12, & i=\frac{n}{2} \text { and } n \text { is even } \\
12(n+i)-11, & \left\lfloor\frac{n+3}{2}\right\rfloor \leq i \leq n\end{cases} \\
& f\left(b_{3, i}\right)= \begin{cases}12(n+i)-5, & 1 \leq i \leq\left\lfloor\frac{n-3}{2}\right\rfloor \\
18 n-10, & i=\frac{n-1}{2} \text { and } n \text { is odd } \\
18 n-16, & i=\frac{n-2}{2} \text { and } n \text { is even } \\
12(n+i)-3, & \left\lfloor\frac{n+1}{2}\right\rfloor \leq i \leq n\end{cases} \\
& f\left(b_{4, i}\right)= \begin{cases}12(n+i)-7, & 1 \leq i \leq\left\lfloor\frac{n-1}{2}\right\rfloor \\
12(n+i)-5, & \left\lfloor\frac{n+1}{2}\right\rfloor \leq i \leq n\end{cases} \\
& f(z)= \begin{cases}18 n-4, & \text { if } n \text { is odd, } \\
18 n-10, & \text { if } n \text { is even }\end{cases} \\
& \text { and } f\left(y_{i}\right)= \begin{cases}12(n+i), & 1 \leq i \leq\left\lfloor\frac{n-3}{2}\right\rfloor \\
18 n-9, & i=\frac{n-1}{2} \text { and } n \text { is odd } \\
18 n-15, & i=\frac{n-2}{2} \text { and } n \text { is even } \\
12(n+i)+2, & \left\lfloor\frac{n+1}{2}\right\rfloor \leq i \leq n-1\end{cases}
\end{aligned}
$$

For the vertex labeling $f$, the induced edge labels are obtained as follows:

$$
\begin{aligned}
f^{*}\left(a_{1, i} a_{2, i}\right) & = \begin{cases}2, & i=1, \\
12(i-1), & 2 \leq i \leq n,\end{cases} \\
f^{*}\left(a_{2, i} u_{i}\right) & = \begin{cases}4, & i=1, \\
12 i-9, & 2 \leq i \leq n,\end{cases} \\
f^{*}\left(a_{3, i} a_{4, i}\right) & =12 i-4, \quad 1 \leq i \leq n, \\
f^{*}\left(a_{4, i} u_{i}\right) & =12 i-6, \quad 1 \leq i \leq n, \\
f^{*}\left(u_{i} x_{i}\right) & =12 i-2, \quad 1 \leq i \leq n-1, \\
f^{*}\left(x_{i} u_{i+1}\right) & =12 i+4, \quad 1 \leq i \leq n-1, \\
f^{*}\left(b_{1, i} b_{2, i}\right) & = \begin{cases}12 n, & i=1, \\
12(n+i)-14, & 2 \leq i \leq\left\lfloor\frac{n-1}{2}\right\rfloor, \\
18 n-7, & i=\frac{n+1}{2} \text { and } n \text { is odd } n \text { is even, } \\
12(n+i)-12, & \left\lfloor\frac{n+3}{2}\right\rfloor \leq i \leq n,\end{cases}
\end{aligned}
$$




$$
\begin{aligned}
& f^{*}\left(b_{2, i} v_{i}\right)= \begin{cases}12 n+2, & i=1, \\
12(n+i)-11, & 2 \leq i \leq\left\lfloor\frac{n-1}{2}\right\rfloor, \\
12(n+i)-9, & \left\lfloor\frac{n+1}{2}\right\rfloor \leq i \leq n,\end{cases} \\
& f^{*}\left(b_{3, i} b_{4, i}\right)= \begin{cases}12(n+i)-6, & 1 \leq i \leq\left\lfloor\frac{n-3}{2}\right\rfloor, \\
18 n-11, & i=\frac{n-1}{2} \text { and } n \text { is odd, } \\
18 n-17, & i=\frac{n-2}{2} \text { and } n \text { is even, } \\
12(n+i)-4, & \left\lfloor\frac{n+1}{2}\right\rfloor \leq i \leq n,\end{cases} \\
& f^{*}\left(b_{4, i} v_{i}\right)= \begin{cases}12(n+i)-8, & 1 \leq i \leq\left\lfloor\frac{n-1}{2}\right\rfloor, \\
12(n+i)-6, & \left\lfloor\frac{n+1}{2}\right\rfloor \leq i \leq n,\end{cases} \\
& f^{*}\left(v_{i} y_{i}\right)= \begin{cases}12(n+i)-4, & 1 \leq i \leq\left\lfloor\frac{n-3}{2}\right\rfloor, \\
18 n-12, & i=\frac{n-1}{2} \text { and } n \text { is odd, } \\
18 n-18, & i=\frac{n-2}{2} \text { and } n \text { is even, } \\
12(n+i)-2, & \left\lfloor\frac{n+1}{2}\right\rfloor \leq i \leq n-1,\end{cases} \\
& \text { and } f^{*}\left(z v_{\frac{n}{2}}\right)=18 n-8 \text { if } n \text { is even. }
\end{aligned}
$$

Thus, $f$ is a super mean labeling and hence $S\left(H_{n} \odot S_{2}\right)$ is a super mean graph. For example, a super mean labeling of $S\left(H_{7} \odot S_{2}\right)$ and $S\left(H_{8} \odot S_{2}\right)$ are shown in Figure 4.

Theorem 2.4. The graph $S\left(S L_{n}\right)$ is a super mean graph, for $n \geq 2$.

Proof. Let $u_{1}, u_{2}, \ldots, u_{n}$ and $v_{1}, v_{2}, \ldots, v_{n}$ be the vertices on the paths of length $n-1$. Let $x_{i}, y_{i}$ and $z_{i}$ be the vertices subdivided the edges $u_{i} u_{i+1}, v_{i} v_{i+1}$ and $v_{i} u_{i+1}$ respectively for each $i, 1 \leq i \leq n-1$. The graph $S\left(S L_{n}\right)$ has $5 n-3$ vertices and $6 n-6$ edges.

Case (i): $n$ is odd. 


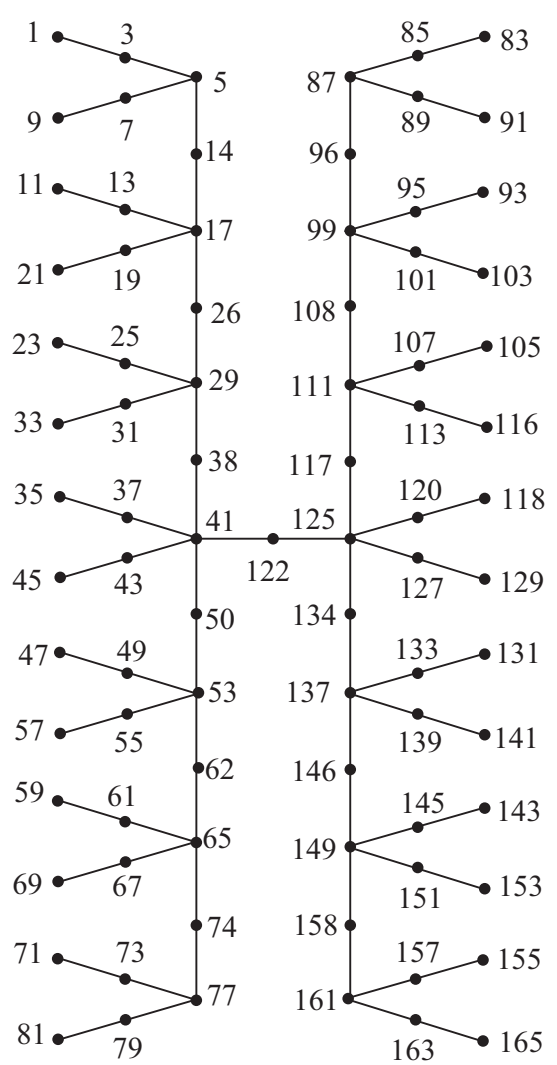

(a) $S\left(H_{7} \odot S_{2}\right)$

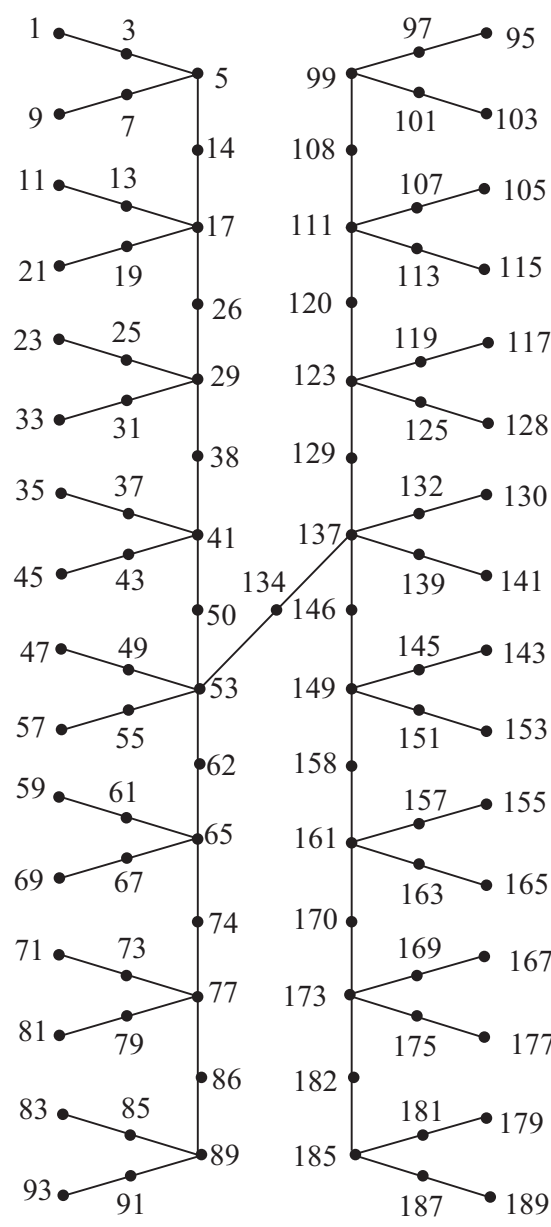

(b) $S\left(H_{8} \odot S_{2}\right)$

Figure 4

Define $f: V\left(S\left(S L_{n}\right)\right) \rightarrow\{1,2, \ldots, p+q=11 n-9\}$ as follows:

$$
\begin{aligned}
& f\left(u_{i}\right)= \begin{cases}1, & i=1, \\
5, & i=2, \\
13, & i=3, \\
11 i-13, & 4 \leq i \leq n \text { and } i \text { is even, } \\
11 i-19, & 4 \leq i \leq n \text { and } i \text { is odd, }\end{cases} \\
& f\left(v_{i}\right)= \begin{cases}11, & i=1, \\
11 i-2, & 2 \leq i \leq n-1 \text { and } i \text { is even, } \\
11 i-8, & 2 \leq i \leq n-1 \text { and } i \text { is odd, } \\
11 n-9, & i=n,\end{cases}
\end{aligned}
$$




$$
\begin{aligned}
f\left(x_{i}\right) & = \begin{cases}3, & i=1, \\
10, & i=2, \\
11 i-5, & 3 \leq i \leq n-1 \text { and } i \text { is odd } \\
11 i-10, & 3 \leq i \leq n-1 \text { and } i \text { is even, }\end{cases} \\
f\left(y_{i}\right) & = \begin{cases}11 i+6, & 1 \leq i \leq n-2 \text { and } i \text { is odd } \\
11 i+1, & 1 \leq i \leq n-2 \text { and } i \text { is even, }\end{cases} \\
f\left(y_{n-1}\right) & =11(n-1), \\
f\left(z_{i}\right) & = \begin{cases}7, & i=1, \\
11 i-6, & 2 \leq i \leq n-1 .\end{cases}
\end{aligned}
$$

For the vertex labeling $f$, the induced edge labeling $f^{*}$ is given follows:

$$
\begin{aligned}
& f^{*}\left(u_{i} x_{i}\right)= \begin{cases}2, & i=1, \\
8, & i=2, \\
11 i-12, & 3 \leq i \leq n-2 \text { and } i \text { is odd } \\
11 i-11, & 3 \leq i \leq n-2 \text { and } i \text { is even, }\end{cases} \\
& f^{*}\left(x_{i} u_{i+1}\right)= \begin{cases}4, & i=1, \\
12, & i=2, \\
11 i-3, & 3 \leq i \leq n-1 \text { and } i \text { is odd, } \\
11 i-9, & 3 \leq i \leq n-1 \text { and } i \text { is even, }\end{cases} \\
& f^{*}\left(v_{i} y_{i}\right)= \begin{cases}14, & i=1, \\
11 i, & 2 \leq i \leq n-2 \text { and } i \text { is even, } \\
11 i-1, & 2 \leq i \leq n-2 \text { and } i \text { is odd, } \\
11 n-12, & i=n-1,\end{cases} \\
& f^{*}\left(y_{i} v_{i+1}\right)= \begin{cases}11 i+8, & 1 \leq i \leq n-2 \text { and } i \text { is odd }, \\
11 i+2, & 1 \leq i \leq n-2 \text { and } i \text { is even, } \\
11 n-10, & i=n-1,\end{cases} \\
& f^{*}\left(v_{i} z_{i}\right)= \begin{cases}9, & i=1, \\
11 i-4, & 2 \leq i \leq n-1 \text { and } i \text { is even, } \\
11 i-6, & 2 \leq i \leq n-1 \text { and } i \text { is odd, }\end{cases} \\
& f^{*}\left(z_{i} u_{i+1}\right)= \begin{cases}6, & i=1, \\
11 i-7, & 2 \leq i \leq n-1 \text { and } i \text { is even, } \\
11 i-4, & 2 \leq i \leq n-1 \text { and } i \text { is odd. }\end{cases}
\end{aligned}
$$

Case (ii): $n$ is even, $n \geq 4$. 
Define $f: V\left(S\left(S L_{n}\right)\right) \rightarrow\{1,2, \ldots, p+q=11 n-9\}$ as follows:

$$
\begin{aligned}
& f\left(u_{i}\right)= \begin{cases}1, & i=1, \\
5, & i=2, \\
13, & i=3, \\
11 i-13, & 4 \leq i \leq n-1 \text { and } i \text { is even, } \\
11 i-19, & 4 \leq i \leq n-1 \text { and } i \text { is odd }, \\
11 n-11, & i=n,\end{cases} \\
& f\left(v_{i}\right)= \begin{cases}11, & i=1, \\
11 i-2, & 2 \leq i \leq n-1 \text { and } i \text { is even, } \\
11 i-8, & 2 \leq i \leq n-1 \text { and } i \text { is odd. } \\
11 n-9, & i=n,\end{cases} \\
& f\left(x_{i}\right)= \begin{cases}3, & i=1, \\
10, & i=2, \\
11 i-5, & 3 \leq i \leq n \text { and } i \text { is odd, } \\
11 i-10, & 3 \leq i \leq n \text { and } i \text { is even, }\end{cases} \\
& f\left(y_{i}\right)= \begin{cases}11 i+6, & 1 \leq i \leq n-2 \text { and } i \text { is odd }, \\
11 i+1, & 1 \leq i \leq n-2 \text { and } i \text { is even, } \\
11 n-12, & i=n-1,\end{cases} \\
& f\left(z_{i}\right)= \begin{cases}7, & i=1, \\
11 i-6, & 2 \leq i \leq n-1 .\end{cases}
\end{aligned}
$$

The induced edge labeling is obtained as follows:

$$
\begin{aligned}
& f^{*}\left(u_{i} x_{i}\right)= \begin{cases}2, & i=1, \\
8, & i=2, \\
11 i-12, & 3 \leq i \leq n-1 \text { and } i \text { is odd } \\
11 i-10, & 3 \leq i \leq n-1 \text { and } i \text { is even, }\end{cases} \\
& f^{*}\left(x_{i} u_{i+1}\right)= \begin{cases}4, & i=1, \\
12, & i=2, \\
11 i-3, & 3 \leq i \leq n-2 \text { and } i \text { is odd } \\
11 i-9, & 3 \leq i \leq n-2 \text { and } i \text { is even, } \\
11 n-13, & i=n-1,\end{cases} \\
& f^{*}\left(v_{i} y_{i}\right)= \begin{cases}14, & i=1, \\
11 i, & 2 \leq i \leq n-2 \text { and } i \text { is even, } \\
11 i-1, & 2 \leq i \leq n-2 \text { and } i \text { is odd } \\
11 n-15, & i=n-1,\end{cases}
\end{aligned}
$$




$$
\begin{aligned}
& f^{*}\left(y_{i} v_{i+1}\right)= \begin{cases}11 i+8, & 1 \leq i \leq n-2 \text { and } i \text { is odd } \\
11 i+2, & 1 \leq i \leq n-2 \text { and } i \text { is even, } \\
11 n-10, & i=n-1,\end{cases} \\
& f^{*}\left(v_{i} z_{i}\right)= \begin{cases}9, & i=1 \\
11 i-4, & 2 \leq i \leq n-1 \text { and } i \text { is even, } \\
11 i-7, & 2 \leq i \leq n-1 \text { and } i \text { is odd }\end{cases} \\
& f^{*}\left(z_{i} u_{i+1}\right)= \begin{cases}6, & i=1, \\
11 i-7, & 2 \leq i \leq n-2 \text { and } i \text { is even, } \\
11 i+7, & 2 \leq i \leq n-2 \text { and } i \text { is odd }, \\
11 n-14, & i=n-1 .\end{cases}
\end{aligned}
$$

Thus, $f$ is a super mean labeling of $S\left(S L_{n}\right)$ and hence $S\left(S L_{n}\right)$ is a super mean graph.

For example, a super mean labeling of $S\left(S L_{7}\right)$ and $S\left(S L_{8}\right)$ are shown in Figure 5.

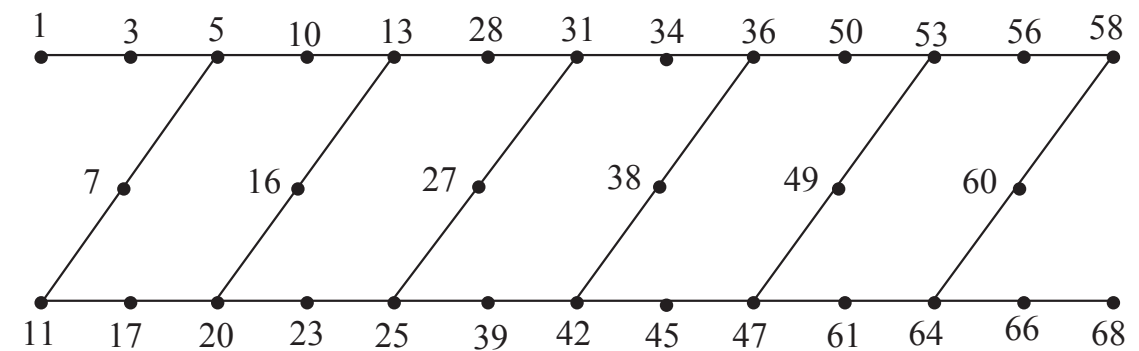

(a) $S\left(S L_{7}\right)$

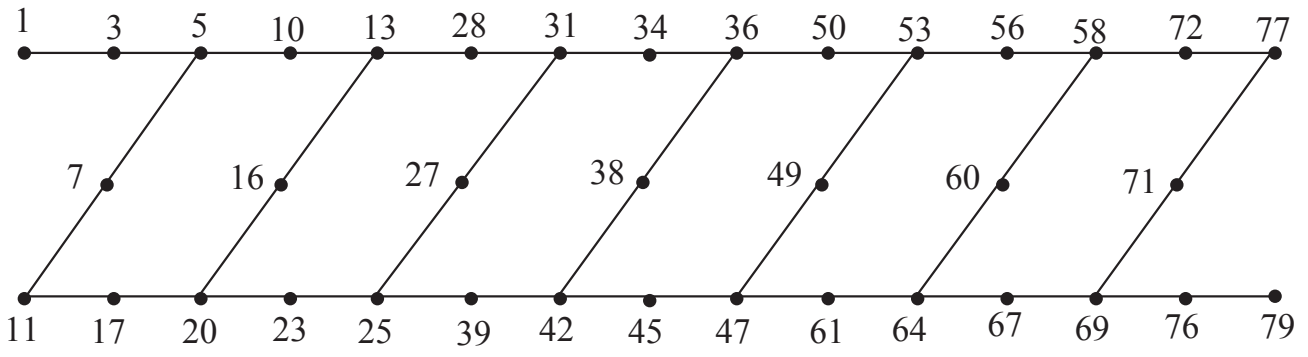

(b) $S\left(S L_{8}\right)$

FiguRE 5

When $n=2$, a super mean labeling of the graph is shown in Figure 6 .

Theorem 2.5. The graph $S\left(T_{n} \odot K_{1}\right)$ is a super mean graph for any $n$.

Proof. Let $u_{1}, u_{2}, \ldots, u_{n}, u_{n+1}$ be the vertices on the path of length $n$ in $T_{n}$ and let $v_{i}$, $1 \leq i \leq n$ be the vertices of $T_{n}$ in which $v_{i}$ is adjacent to $u_{i}$ and $u_{i+1}$. Let $v_{i}^{\prime} a_{i} v_{i}$ be the path attached at each $v_{i}, 1 \leq i \leq n$ and $u_{i}^{\prime} b_{i} u_{i}$ be the path attached at each $u_{i}$, $1 \leq i \leq n+1$. Let $x_{i}, y_{i}$ and $z_{i}$ be the vertices which subdivided the edges $u_{i} u_{i+1}, u_{i} v_{i}$ 


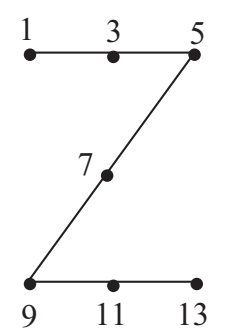

FIGURE 6

and $v_{i} u_{i+1}$ respectively for each $i, 1 \leq i \leq n$. The graph $S\left(T_{n} \odot K_{1}\right)$ has $9 n+3$ vertices and $10 n+2$ edges.

Define $f: V\left(S\left(T_{n} \odot K_{1}\right)\right) \rightarrow\{1,2, \ldots, p+q=19 n+5\}$ as follows:

$$
\begin{aligned}
& f\left(u_{i}\right)=19 i-14, \quad 1 \leq i \leq n+1, \\
& f\left(v_{i}\right)=19 i-8, \quad 1 \leq i \leq n, \\
& f\left(v_{i}^{\prime}\right)=19 i-4, \quad 1 \leq i \leq n, \\
& f\left(a_{i}\right)=19 i-6, \quad 1 \leq i \leq n, \\
& f\left(u_{i}^{\prime}\right)= \begin{cases}1, & i=1, \\
19 i-20, & 2 \leq i \leq n+1,\end{cases} \\
& f\left(b_{i}\right)= \begin{cases}3, & i=1, \\
19 i-18, & 2 \leq i \leq n+1,\end{cases} \\
& f\left(x_{i}\right)=19 i-9, \quad 1 \leq i \leq n, \\
& f\left(y_{i}\right)=19 i-12, \quad 1 \leq i \leq n, \\
& f\left(z_{i}\right)=19 i+2, \quad 1 \leq i \leq n .
\end{aligned}
$$

The induced edge labeling is defined as follows:

$$
\begin{aligned}
f^{*}\left(u_{i} x_{i}\right) & =19 i-11, \quad 1 \leq i \leq n, \\
f^{*}\left(x_{i} u_{i+1}\right) & =19 i-2, \quad 1 \leq i \leq n, \\
f^{*}\left(u_{i} y_{i}\right) & =19 i-13, \quad 1 \leq i \leq n, \\
f^{*}\left(y_{i} v_{i}\right) & =19 i-10, \quad 1 \leq i \leq n, \\
f^{*}\left(v_{i} z_{i}\right) & =19 i-3, \quad 1 \leq i \leq n, \\
f^{*}\left(z_{i} u_{i+1}\right) & =19 i+4, \quad 1 \leq i \leq n, \\
f^{*}\left(v_{i} a_{i}\right) & =19 i-7, \quad 1 \leq i \leq n, \\
f^{*}\left(a_{i} v_{i}^{\prime}\right) & =19 i-5, \quad 1 \leq i \leq n, \\
f^{*}\left(u_{i} b_{i}\right) & =\left\{\begin{array}{lr}
4, & i=1, \\
19 i-16, & 2 \leq i \leq n+1,
\end{array}\right.
\end{aligned}
$$




$$
f^{*}\left(b_{i} u_{i}^{\prime}\right)= \begin{cases}2, & i=1 \\ 19(i-1), & 2 \leq i \leq n+1 .\end{cases}
$$

Thus, $f$ is a super mean labeling of $S\left(T_{n} \odot K_{1}\right)$.

For example, a super mean labeling of $S\left(T_{6} \odot K_{1}\right)$ is shown in Figure 7.

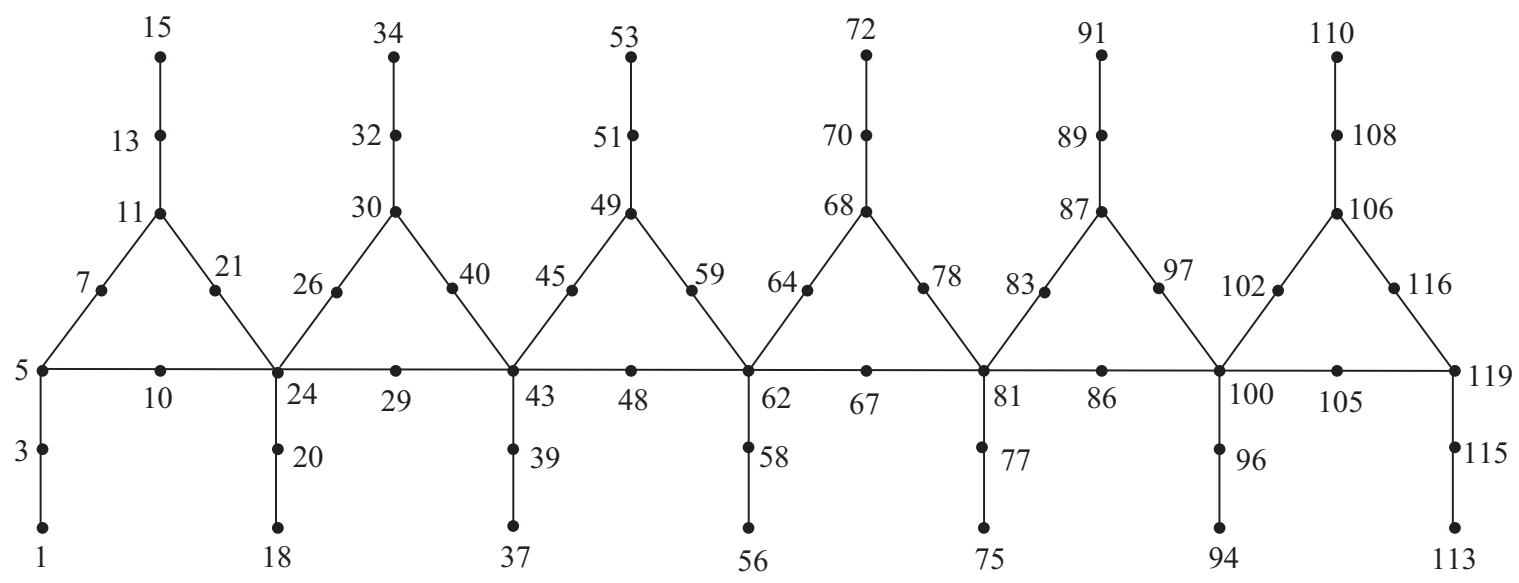

FiguRE 7. $S\left(T_{6} \odot K_{1}\right)$

Theorem 2.6. The graph $S\left(C_{n} \odot K_{1}\right)$ is a super mean graph, for $n \geq 3$.

Proof. Let $u_{1}, u_{2}, \ldots, u_{n}$ be the vertices of the cycle $C_{n}$. Let $v_{i} y_{i} u_{i}$ be the path attached at each $u_{i}, 1 \leq i \leq n$. Each edge $u_{i} u_{i+1}$ is subdivided by a vertex $x_{i}, 1 \leq i \leq n-1$ and the edge $u_{n} u_{1}$ is subdivided by a vertex $x_{n}$.

Case(i): $n$ is odd.

Define $f: V\left(S\left(C_{n} \odot K_{1}\right)\right) \rightarrow\{1,2, \ldots, 8 n\}$ as follows:

$$
\begin{aligned}
& f\left(u_{i}\right)= \begin{cases}5, & i=1, \\
16 i-21, & 2 \leq i \leq \frac{n+1}{2}, \\
8 n, & i=\frac{n+3}{2}, \\
16(n-i)+22, & \frac{n+5}{2} \leq i \leq n,\end{cases} \\
& f\left(v_{i}\right)= \begin{cases}1, & i=1 \\
16 i-17, & 2 \leq i \leq \frac{n+1}{2} \\
16(n-i)+18, & \frac{n+3}{2} \leq i \leq n,\end{cases} \\
& f\left(x_{i}\right)= \begin{cases}16 i-9, & 1 \leq i \leq \frac{n-1}{2} \\
8 n-3, & i=\frac{n+1}{2} \\
16(n-i)+10, & \frac{n+3}{2} \leq i \leq n,\end{cases}
\end{aligned}
$$




$$
f\left(y_{i}\right)= \begin{cases}3, & i=1 \\ 16 i-19, & 2 \leq i \leq \frac{n+1}{2} \\ 16(n-i)+20, & \frac{n+3}{2} \leq i \leq n\end{cases}
$$

The induced edge labeling is defined as follows:

$$
\begin{aligned}
& f^{*}\left(u_{i} x_{i}\right)= \begin{cases}6, & i=1, \\
16 i-15, & 2 \leq i \leq \frac{n-1}{2}, \\
8(n-1), & i=\frac{n+1}{2}, \\
8 n-7, & i=\frac{n+3}{2}, \\
16(n-i)+16, & \frac{n+5}{2} \leq i \leq n,\end{cases} \\
& f^{*}\left(x_{i} u_{i+1}\right)= \begin{cases}16 i-7, & 1 \leq i \leq \frac{n-1}{2}, \\
8 n-1, & i=\frac{n+1}{2}, \\
16(n-i)+8, & \frac{n+3}{2} \leq i \leq n-1,\end{cases} \\
& f^{*}\left(x_{n} u_{1}\right)=8, \\
& f^{*}\left(v_{i} y_{i}\right)= \begin{cases}2, & i=1, \\
16 i-18, & 2 \leq i \leq \frac{n+1}{2}, \\
16(n-i)+19, & \frac{n+3}{2} \leq i \leq n,\end{cases} \\
& \text { and } f^{*}\left(y_{i} u_{i}\right)= \begin{cases}4, & i=1, \\
16 i-20, & 2 \leq i \leq \frac{n+1}{2}, \\
8 n-2, & i=\frac{n+3}{2}, \\
16(n-i)+21, & \frac{n+5}{2} \leq i \leq n .\end{cases}
\end{aligned}
$$

Case (ii): $n$ is even.

$$
\begin{gathered}
f\left(u_{i}\right)= \begin{cases}5, & i=1, \\
16 i-21, & 2 \leq i \leq \frac{n}{2}, \\
8 n-4, & i=\frac{n+2}{2}, \\
16(n-i)+22, & \frac{n+4}{2} \leq i \leq n,\end{cases} \\
f\left(v_{i}\right)= \begin{cases}1, & i=1, \\
16 i-17, & 2 \leq i \leq \frac{n}{2}, \\
8 n, & i=\frac{n+2}{2}, \\
16(n-i)+18, & \frac{n+4}{2} \leq i \leq n,\end{cases} \\
f\left(x_{i}\right)= \begin{cases}16 i-9, & 1 \leq i \leq \frac{n}{2}, \\
8 n-7, & i=\frac{n+2}{2}, \\
16(n-i)+10, & \frac{n+4}{2} \leq i \leq n,\end{cases}
\end{gathered}
$$




$$
f\left(y_{i}\right)= \begin{cases}3, & i=1 \\ 16 i-19, & 2 \leq i \leq \frac{n}{2} \\ 8 n-2, & i=\frac{n+2}{2} \\ 16(n-i)+20, & \frac{n+4}{2} \leq i \leq n\end{cases}
$$

For the vertex labeling $f$, the induced edge labeling $f^{*}$ is given as follows:

$$
\begin{aligned}
& f^{*}\left(u_{i} x_{i}\right)= \begin{cases}6, & i=1, \\
16 i-15, & 2 \leq i \leq \frac{n}{2}, \\
8 n-5, & i=\frac{n+2}{2}, \\
16(n-i+1), & \frac{n+4}{2} \leq i \leq n,\end{cases} \\
& f^{*}\left(x_{i} u_{i+1}\right)= \begin{cases}16 i-7, & 1 \leq i \leq \frac{n-2}{2}, \\
8 n-6, & i=\frac{n}{2}, \\
16(n-i)+8, & \frac{n+2}{2} \leq i \leq n-1,\end{cases} \\
& f^{*}\left(x_{n} u_{1}\right) 8, \\
& f^{*}\left(v_{i} y_{i}\right)= \begin{cases}2, & i=1, \\
16 i-18, & 2 \leq i \leq \frac{n}{2}, \\
8 n-1, & i=\frac{n+2}{2}, \\
16(n-i)+19, & \frac{n+4}{2} \leq i \leq n,\end{cases} \\
& \text { and } f^{*}\left(y_{i} u_{i}\right)= \begin{cases}4, & i=1, \\
16 i-20, & 2 \leq i \leq \frac{n}{2}, \\
8 n-3, & i=\frac{n+2}{2}, \\
16(n-i)+21, & \frac{n+4}{2} \leq i \leq n .\end{cases}
\end{aligned}
$$

Thus, $f$ is a super mean labeling and hence $S\left(C_{n} \odot K_{1}\right)$ is a super mean graph.

For example, a super mean labeling of $S\left(C_{11} \odot K_{1}\right)$ and $S\left(C_{12} \odot K_{1}\right)$ are shown in Figure 8.

Theorem 2.7. The graph $S\left(C_{m} @ C_{n}\right)$ is a super mean graph for $m, n \geq 3$.

Proof. $C_{m} @ C_{n}$ is a graph obtained by identifying an edge of two cycles $C_{m}$ and $C_{n}$. $C_{m} @ C_{n}$ has $m+n-2$ vertices and $m+n-1$ edges. In $S\left(C_{m} @ C_{n}\right), 2(m+n-2)$ vertices lies on the circle and one vertex lies on a chord. Then, the graph $S\left(C_{m} @ C_{n}\right)$ has $2 m+2 n-3$ vertices and $2(m+n-1)$ edges.

Let us assume that $m \leq n$. 


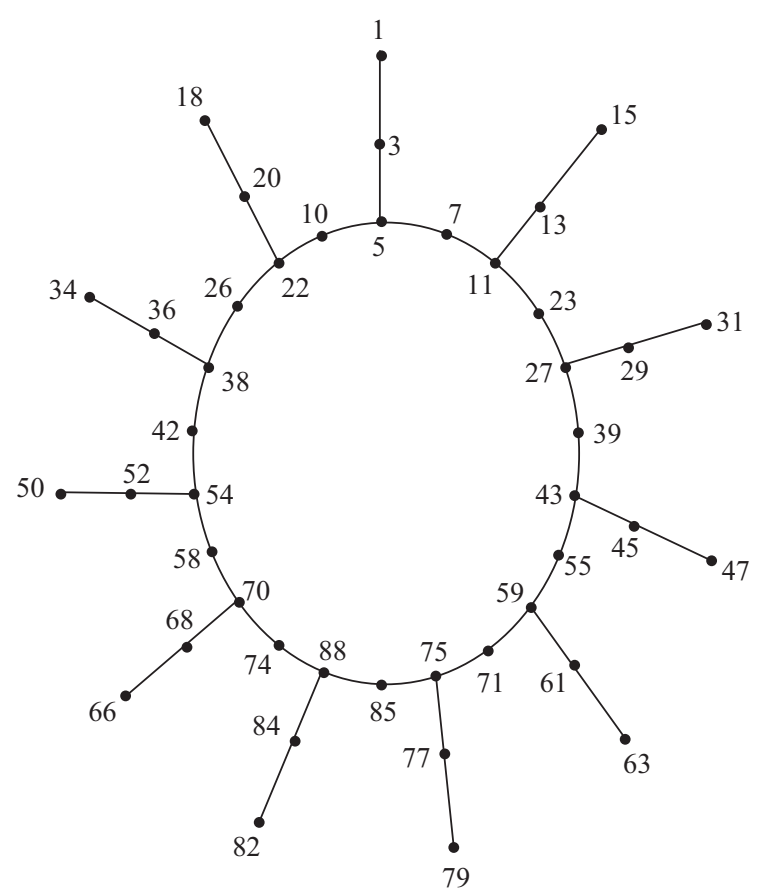

(a) $S\left(C_{11} \odot K_{1}\right)$

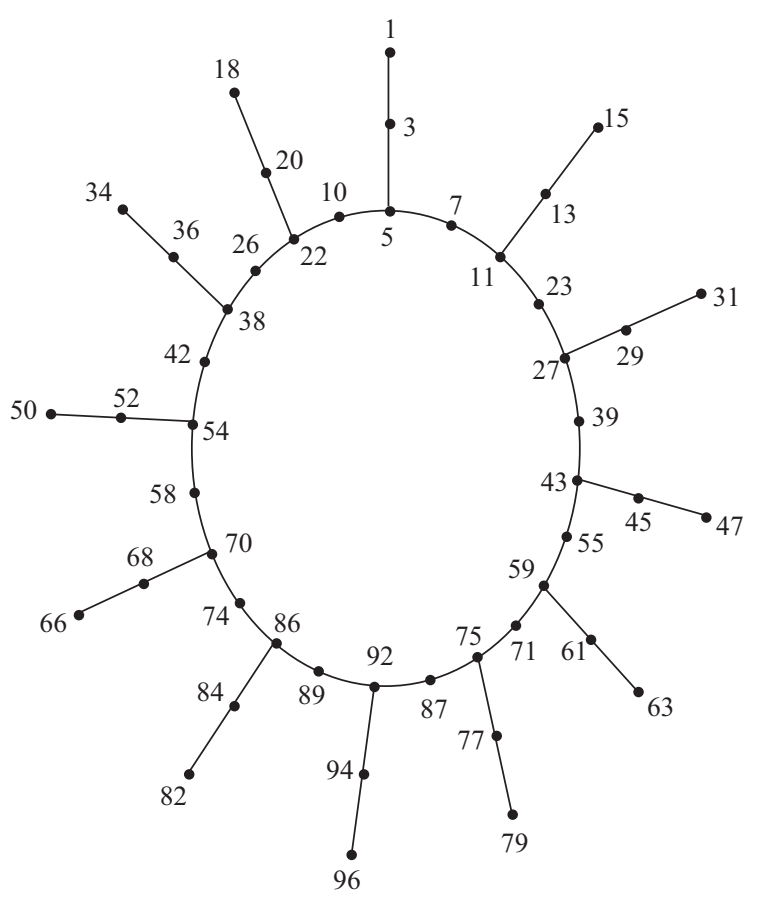

(b) $S\left(C_{12} \odot K_{1}\right)$

FiguRe 8

Case (i): $m$ is odd and $n$ is odd.

Let $m=2 k+1, k \geq 1$ and $n=2 l+1, l \geq 1$. We denote the vertices of $S\left(C_{m} @ C_{n}\right)$ is shown in Figure 9.

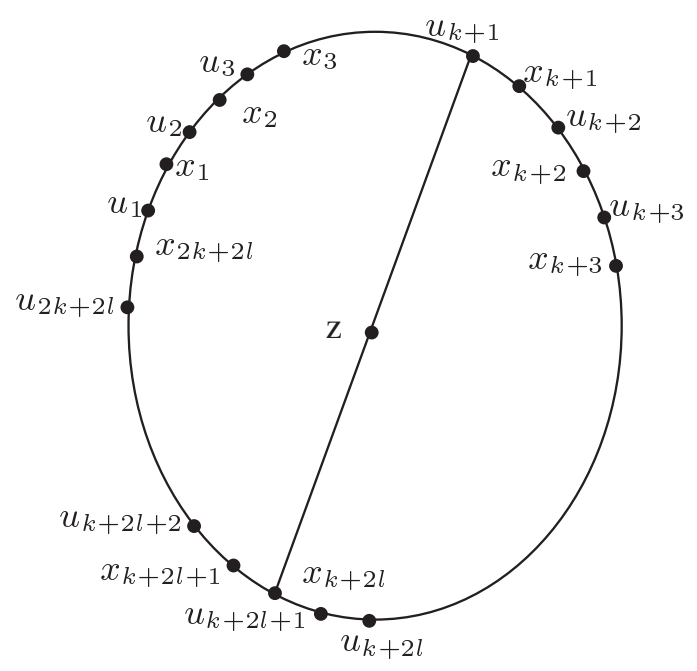

FiguRE 9 
Define $f: V\left(S\left(C_{m} @ C_{n}\right)\right) \rightarrow\{1,2,3, \ldots, p+q=4(m+n)-5\}$ as follows:

$$
\begin{aligned}
& f\left(u_{i}\right)= \begin{cases}1, & i=1, \\
8 i-9, & 2 \leq i \leq k, \\
4 m-6, & i=k+1, \\
8 i, & k+2 \leq i \leq k+l, \\
8(m+n-i)-9, & k+l+1 \leq i \leq k+2 l-1, \\
4 m+5, & i=k+2 l, \\
4 m, & i=k+2 l+1, \\
8(m+n-i)-6, & k+2 l+2 \leq i \leq 2 k+2 l,\end{cases} \\
& f\left(x_{i}\right)= \begin{cases}8 i-5, & 1 \leq i \leq k, \\
8 i+4, & k+1 \leq i \leq k+l-1, \\
8(m+n-i)-13, & k+l \leq i \leq k+2 l-1, \\
4 m+3, & i=k+2 l, \\
4 m-5, & i=k+2 l+1, \\
8(m+n-i)-10, & k+2 l+2 \leq i \leq 2 k+2 l,\end{cases}
\end{aligned}
$$

and $f(z)=4 m-3$.

The induced edge labeling $f^{*}$ is obtained as follows:

$$
\begin{aligned}
f^{*}\left(u_{i} x_{i}\right)= & \begin{cases}2, & i=1, \\
8 i-7, & 2 \leq i \leq k, \\
4 m+1, & i=k+1, \\
8 i+2, & k+2 \leq i \leq k+l, \\
8(m+n-i)-11, & k+l+1 \leq i \leq k+2 l-1, \\
4 m+4, & i=k+2 l, \\
4 m-2, & i=k+2 l+1, \\
8(m+n-1-i), & k+2 l+2 \leq i \leq 2 k+2 l-2, \\
8 i-3, & 1 \leq i \leq k, \\
8 i+6, & k+1 \leq i \leq k+l-1, \\
8(m+n-i)-15, & k+l \leq i \leq k+2 l-2, \\
4 m+6, & i=k+2 l-1, \\
4 m+2, & i=k+2 l, \\
8(m+n-i)-12, & k+2 l+1 \leq i \leq 2 k+2 l-1,\end{cases} \\
f^{*}\left(x_{i} u_{i+1}\right)= & \\
f^{*}\left(x_{2 k+2 l} u_{1}\right)= & \\
f^{*}\left(u_{k+1} z\right)=4 m-4, &
\end{aligned}
$$


Thus, $f$ is a super mean labeling. A super mean labeling of $S\left(C_{7} @ C_{9}\right)$ is shown in Figure 10.

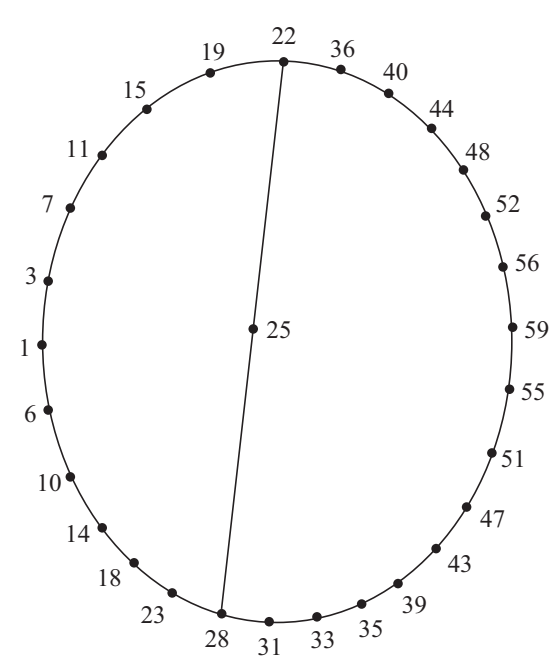

FiguRE 10

Case (ii): $m$ is odd and $n$ is even.

Let $m=2 k+1, k \geq 1$ and $n=2 l, l \geq 2$.

Define $f: V\left(S\left(C_{m} @ C_{n}\right)\right) \rightarrow\{1,2,3, \ldots, p+q=4(m+n)-5\}$ as follows:

$$
\begin{aligned}
f\left(u_{i}\right)= \begin{cases}1, & i=1 \\
8 i-9, & 2 \leq i \leq k \\
4 m-6, & i=k+1 \\
8 i, & k+2 \leq i \leq k+l-1 \\
8(m+n-i)-9, & k+l \leq i \leq k+2 l-2, \\
4 m+5, & i=k+2 l-1 \\
4 m, & i=k+2 l \\
8(m+n-i)-6, & k+2 l+1 \leq i \leq 2 k+2 l-1,\end{cases} \\
f\left(x_{i}\right)= \begin{cases}8 i-5, & 1 \leq i \leq k \\
8 i+4, & k+1 \leq i \leq k+l-1, \\
8(m+n-i)-13, & k+l \leq i \leq k+2 l-2, \\
4 m+3, & i=k+2 l-1 \\
4 m-5, & i=k+2 l \\
8(m+n-i)-10, & k+2 l+1 \leq i \leq 2 k+2 l-1,\end{cases}
\end{aligned}
$$

and $f(z)=4 m-3$. 
For the vertex labeling $f$, the induced edge labeling $f^{*}$ is given as follows:

$$
\begin{aligned}
f^{*}\left(u_{i} x_{i}\right)= & \begin{cases}2, & i=1, \\
8 i-7, & 2 \leq i \leq k, \\
4 m+1, & i=k+1, \\
8 i+2, & k+2 \leq i \leq k+l-1, \\
8(m+n-i)-11, & k+l \leq i \leq k+2 l-2, \\
4 m+4, & i=k+2 l-1, \\
4 m-2, & i=k+2 l, \\
8(m+n-i)-8, & k+2 l+1 \leq i \leq 2 k+2 l-1, \\
8 i-3, & 1 \leq i \leq k, \\
8 i+6, & k+1 \leq i \leq k+l-1, \\
8(m+n-i)-15, & k+l \leq i \leq k+2 l-3, \\
4 m+6, & i=k+2 l-2, \\
4 m+2, & i=k+2 l-1, \\
8(m+n-i)-12, & k+2 l \leq i \leq 2 k+2 l-2,\end{cases} \\
f^{*}\left(x_{i} u_{i+1}\right)= & \\
f^{*}\left(x_{2 k+2 l-1} u_{1}\right)= & \\
f^{*}\left(u_{k+1} z\right)= & 4 m-4, \\
\text { and } f^{*}\left(z u_{k+2 l}\right)= & 4 m-1 .
\end{aligned}
$$

Thus, $f$ is a super mean labeling. A super mean labeling of $S\left(C_{7} @ C_{10}\right)$ is shown in Figure 11.

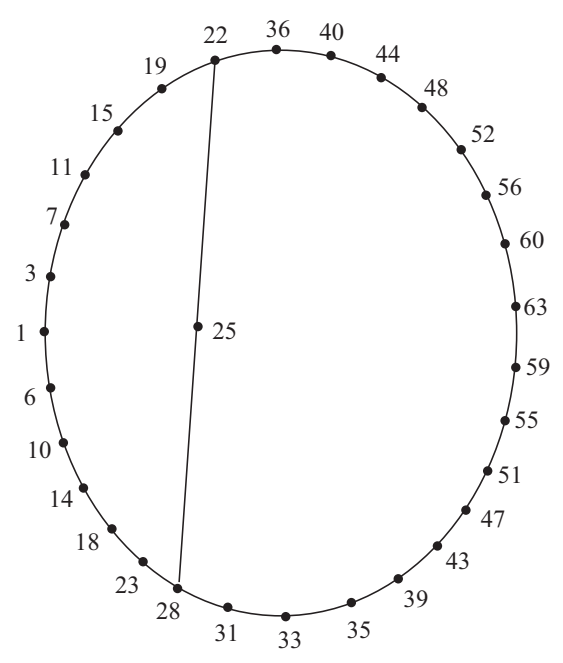

FIGURE 11

Case (iii): $m$ is even and $n$ is even.

Let $m=2 k, k \geq 2$ and $n=2 l, l \geq 2$. 
Define $f: V\left(S\left(C_{m} @ C_{n}\right)\right) \rightarrow\{1,2,3, \ldots, p+q=4(m+n)-5\}$ as follows:

$$
\begin{aligned}
& f\left(u_{i}\right)= \begin{array}{ll}
1, & i=1, \\
8 i-9, & 2 \leq i \leq k, \\
4 m, & i=k+1 \\
4 m+5, & i=k+2, \\
8 i-13, & k+3 \leq i \leq k+l+1, \\
8(m+n-i)+4, & k+l+2 \leq i \leq k+2 l-1, \\
8(m+n-i)-6, & k+2 l \leq i \leq 2 k+2 l-2,
\end{array} \\
& f\left(x_{i}\right)= \begin{cases}8 i-5, & 1 \leq i \leq k+1 \\
8 i-9, & k+2 \leq i \leq k+l, \\
8(m+n-i), & k+l+1 \leq i \leq k+2 l-1, \\
8(m+n-i)-10, & k+2 l \leq i \leq 2 k+2 l-2,\end{cases}
\end{aligned}
$$

and $f(z)=4 m-3$.

For the vertex labeling $f$, the induced edge labeling $f^{*}$ is obtained as follows:

$$
\begin{aligned}
f^{*}\left(u_{i} x_{i}\right)= \begin{cases}2, & i=1, \\
8 i-7, & 2 \leq i \leq k, \\
4 m+2, & i=k+1, \\
4 m+6, & i=k+2, \\
8 i-11, & k+3 \leq i \leq k+l, \\
8(m+n-i)+2, & k+l+1 \leq i \leq k+2 l-1, \\
8(m+n-i)-8, & k+2 l \leq i \leq 2 k+2 l-2,\end{cases} \\
f^{*}\left(x_{i} u_{i+1}\right)= \begin{cases}8 i-3, & 1 \leq i \leq k-1, \\
4 m-2, & i=k, \\
4 m+4, & i=k+1, \\
8(m+n-i)-2, & k+2 \leq i \leq k+l, \\
4 m+1, & i=k+2 l-1, \\
8(m+n-i)-12, & k+2 l \leq i \leq 2 k+2 l-3,\end{cases} \\
f^{*}\left(x_{2 k+2 l-2} u_{1}\right)=4, \\
f^{*}\left(u_{k+1} z\right)=4 m-1, \\
\text { and } f^{*}\left(z u_{k+2 l}\right)=4 m-4 .
\end{aligned}
$$

Thus, $f$ is a super mean labeling. A super mean labeling of $S\left(C_{6} @ C_{8}\right)$ is shown in Figure 12.

Hence, the graph $S\left(C_{m} @ C_{n}\right)$ is a super mean graph for $m, n \geq 3$. 


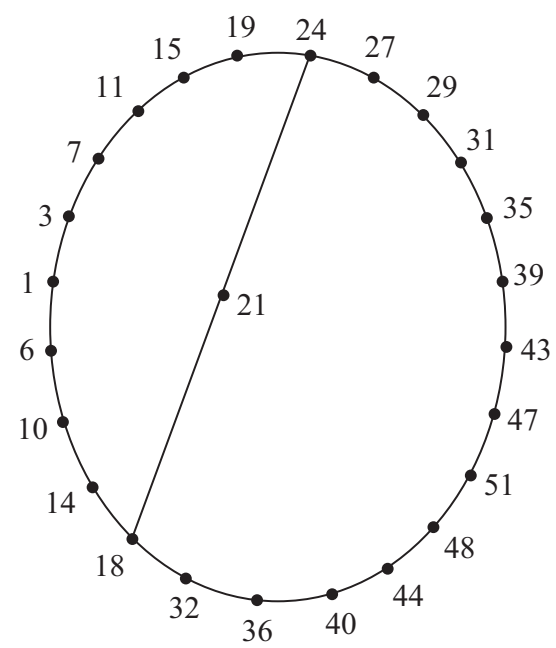

FIGURE 12

\section{REFERENCES}

[1] J. A. Gallian, A dynamic survey of graph labeling, The Electronic Journal of Combinatorics 18 (2015), Article ID \#DS6

[2] F. Harary, Graph Theory, Addison-Wesley, Reading Mass., 1972.

[3] A. Nagarajan, R. Vasuki and S. Arockiaraj, Super mean number of a graph, Kragujevac J. Math. 36(1) (2012), 61-75.

[4] D. Ramya, R. Ponraj and P. Jeyanthi, Super mean labeling of graphs, Ars Combin. 112 (2013), 65-72.

[5] S. Somasundaram and R. Ponraj, Mean labelings of graphs, Nat. Acad. Sci. Lett. 26(7) (2003), $210-213$.

[6] R. Vasuki and A. Nagarajan, Some results on super mean graphs, International J. Math. Combin. 3 (2009), 82-96.

[7] R. Vasuki and A. Nagarajan, On the construction of new classes of super mean graphs, J. Discrete Math. Sci. Cryptogr. 13(3) (2010), 277-290.

[8] R. Vasuki and A. Nagarajan, Further results on super mean graphs, J. Discrete Math. Sci. Cryptogr. 14(2) (2011), 193-206.

[9] R. Vasuki and S. Arockiaraj, On super mean graphs, Util. Math., (in press).

[10] S. K. Vaidya and L. Bijukumar, Some new families of mean graphs, Journal of Mathematics Research 2(3) (2010), 169-176.

[11] S. K. Vaidya and L. Bijukumar, Mean labeling for some new families of graphs, Journal of Pure and Applied Sciences 18 (2010), 115-116.

${ }^{1}$ Department of Mathematics,

Dr. Sivanthi Aditanar College of Engineering

Tiruchendur-628 215, TAMIl NAdU

INDIA

E-mail address: vasukisehar@gmail.com, p.sugisamy28@gmail.com,

revathi198715@gmail.com 